\title{
A summary of observational records on periodicities above the rotational period in the Jovian magnetosphere
}

\author{
E. A. Kronberg, J. Woch, N. Krupp, and A. Lagg \\ Max-Planck-Institut für Sonnensystemforschung, Katlenburg-Lindau, Germany \\ Received: 16 October 2008 - Revised: 15 June 2009 - Accepted: 15 June 2009 - Published: 25 June 2009
}

\begin{abstract}
The Jovian magnetosphere is a very dynamic system. The plasma mass-loading from the moon Io and the fast planetary rotation lead to regular release of mass from the Jovian magnetosphere and to a change of the magnetic topology. These regular variations, most commonly on several (2.5-4) days scale, were derived from various data sets obtained by different spacecraft missions and instruments ranging from auroral images to in situ measurements of magnetospheric particles. Specifically, ion measurements from the Galileo spacecraft represent the periodicities, very distinctively, namely the periodic thinning of the plasma sheet and subsequent dipolarization, and explosive mass release occurring mainly during the transition between these two phases. We present a review of these periodicities, particularly concentrating on those observed in energetic particle data. The most distinct periodicities are observed for ions of sulfur and oxygen. The periodic topological change of the Jovian magnetosphere, the associated mass-release process and auroral signatures can be interpreted as a global magnetospheric instability with analogies to the two step concept of terrestrial substorms. Different views on the triggering mechanism of this magnetospheric instability are discussed.
\end{abstract}

Keywords. Magnetospheric physics (Magnetospheric configuration and dynamics; Planetary magnetospheres)

\section{Introduction}

Quasi-periodic modulations in the Jovian magnetosphere with a repetition period of several days were reported in a number of scientific papers. The first comprehensive investigations were made by using data from the Galileo mission. Periodicities of explosive energetic particle flow bursts with an average repetition period of about 2 to 3 days were revealed in the pre-dawn region of the Jovian magnetotail (Krupp et al., 1998). Louarn et al. (1998) associated these periodicities with the periodic intensification of auroral emissions. These striking observations were made on a single Galileo orbit (G2) which is supposedly closely aligned in the equatorial plane with the Jovian x-line (Woch et al., 2002). These initial reports of the periodic activity associated with energetic events were from a single orbit in the magnetotail. On the other hand periodic thinning and thickening of the plasma sheet with a repetition period of $\sim 3$ days were observed by Woch et al. (1998) along the prolongated trajectory which started in the middle magnetosphere, $\sim 20 R_{J}$ until $\sim 145 R_{J}$.

Are these periodicities a common feature or rather rare events connected with specific external or internal conditions as for example extreme changes in the solar wind? If they are a common feature, are they internally triggered or externally? Is the repetition period of this plasma sheet reconfiguration always close to 2-3 days or are there more variations? Could these periodicities be fitted in a modern concept of a substorm? These are the questions we would like to address in this paper.

The aim is to review evidences for the periodical reconfiguration processes in the Jovian magnetosphere and to show that this dynamics is commonly observed in the Jovian magnetosphere, not only on specific orbits. Therefore, the observed periodicities from different instruments and space missions, will be summarized and extended with yet unpublished data from the Energetic Particle Detector (EPD) on Galileo. 

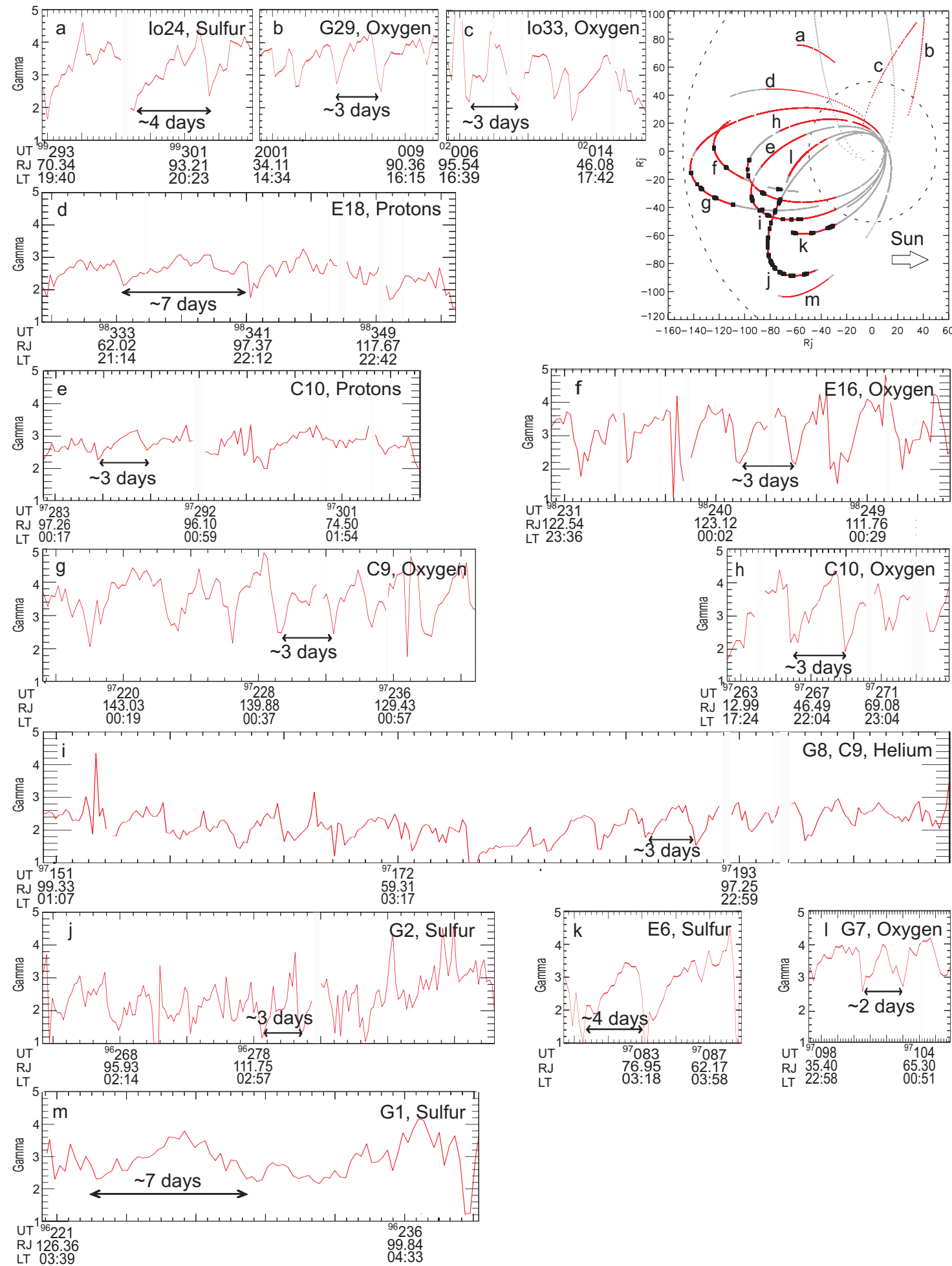

Fig. 1. The G2-Io33 orbits of Galileo in Jupiter Solar Ecliptic coordinates excluding time periods with insufficient data coverage (upper right panel). Along red coloured orbits Galileo observed a periodic behavior of the ion spectral index $\gamma$. The spectral index for protons is calculated using two energy channels (80-220 keV/nuc and 220-540 keV/nuc); for helium (27-155 keV/nuc and 155-1000 keV/nuc); for oxygen (26$51 \mathrm{keV} /$ nuc and 51-112 keV/nuc); for sulfur (16-30 keV/nuc and 30-62 keV/nuc). The locations of the flow bursts, or reconfiguration events (as identified in Woch et al. (1999); Kronberg et al. (2005)) are marked by black dots. These flow bursts are also present in Figs. 2-5, as deviations from the corotational flow. Each time series is denoted by a letter which shows the location of the corresponding orbit (see upper right panel). 
Table 1. Periodicities in the Jovian magnetosphere.

\begin{tabular}{|c|c|c|c|c|c|}
\hline Year & DOY & Orbit & Which type of periodicities & Period & Publication \\
\hline 1996 & $220-238$ & G1 & $\gamma($ for $\mathrm{p}, \mathrm{O}$ and $\mathrm{S})$, magnetic field & 7 days & new \\
\hline 1996 & $223-296$ & G1 & count rates from PLS & $5-7$ days & Vasyliūnas et al. (1997) \\
\hline 1996 & $262-296$ & $\mathrm{G} 2$ & $\begin{array}{l}\text { Anisotropy, magnetic field, } \gamma \text { (for } \mathrm{p}, \mathrm{O} \text { and } \mathrm{S} \text { ), auroral } \\
\text { wave emissions }\end{array}$ & 2.5-3 days & $\begin{array}{l}\text { Krupp et al. (1998); Louarn et al. } \\
\text { (1998); Woch et al. (1998); Kro- } \\
\text { nberg et al. (2007); data partly } \\
\text { published from DOY } 265 \text { to } 280\end{array}$ \\
\hline 1997 & 078-089 & E6 & $\gamma($ for $\mathrm{p}, \mathrm{He}, \mathrm{O}$ and $\mathrm{S})$ auroral wave emissions & 4-5 days & Louarn et al. (2000) \\
\hline 1997 & 097-106 & G7 & $\gamma$ (for $\mathrm{p}, \mathrm{O}$ and $\mathrm{S}$ ), auroral wave emissions & $2-3$ days & Louarn et al. (2000) \\
\hline 1997 & $150-206$ & G8,C9 & $\begin{array}{l}\text { magnetic field, } \gamma \text { (for } \mathrm{p}, \mathrm{He}, \mathrm{O} \text { and } \mathrm{S} \text { ), particle flux, } \\
\text { auroral wave emissions }\end{array}$ & 1.5-4 days & $\begin{array}{l}\text { Woch et al. (1998); Kronberg } \\
\text { et al. (2008a); published to DOY } \\
\text { 200;Louarn et al. (2000) }\end{array}$ \\
\hline 997 & $215-241$ & C9 & $\gamma($ for $\mathrm{p}, \mathrm{He}, \mathrm{O}$ and $\mathrm{S})$ & 3 days & Woch et al. (1998) \\
\hline 1998 & $328-354$ & E18 & $\gamma($ for $\mathrm{p}, \mathrm{He}, \mathrm{O}$ and $\mathrm{S})$ & $2-7$ days & new \\
\hline 1999 & $292-305$ & Io24 & $\gamma($ for $\mathrm{p}, \mathrm{He}, \mathrm{O}$ and $\mathrm{S})$ & 4 days & new \\
\hline 2001 & 000-011 & G29 & $\gamma($ for $\mathrm{p}, \mathrm{He}$, and $\mathrm{O})$ & 3 days & new \\
\hline 2002 & $005-017$ & Io33 & $\gamma($ for $\mathrm{p}$ and $\mathrm{O})$ & 3 days & new \\
\hline \multicolumn{6}{|c|}{ Observations by other experiments } \\
\hline 1996 & $230-269$ & IUE & "auroral activity index" & 5-10 days & Prangé et al. (2001) \\
\hline 2007 & $051-069$ & HST & occurrence of polar dawn spots & $2-3$ days & Radioti et al. (2008) \\
\hline 2007 & 085-104 & $\mathrm{NH}$ & variations in flow speed & 3-4 days & McComas et al. (2007) \\
\hline 2007 & 085-104 & $\mathrm{NH}$ & $\begin{array}{l}\text { velocity dispersions, anisotropies and compositional } \\
\text { variations }\end{array}$ & 3-4 days & McNutt et al. (2007) \\
\hline
\end{tabular}

\section{Observations}

In Fig. 1 we present an overview of the periodicities of the energy spectral index gamma $\gamma$ which is calculated by assuming a power law fit where gamma is the slope of the energy spectra in a log-log plot. The periodicities are observed for protons in the $80-540 \mathrm{keV} /$ nuc energy range, for helium in the $27-1000 \mathrm{keV} /$ nuc, oxygen in the $26-112 \mathrm{keV} /$ nuc energy range and sulfur in the 16-62 keV/nuc energy range by the EPD on Galileo for the time periods listed in Table 1. The Galileo orbits are shown in the right top corner of Fig. 1. The red coloured Galileo orbits show locations where the periodicities of the spectral index were observed. In the present paper we show the time series of the spectral index explicitly, whereas in Kronberg et al. (2007) they were shown only by wavy lines on the plot with Galileo orbits. Further we will review these periodicities.

\subsection{Plasma sheet modulations}

The initial work on periodic changes of the plasma sheet structure was done by Vasyliūnas et al. (1997). They used data from Galileo Plasma Subsystem Instrumentation (PLS) and reported a 5-7 day modulation of the electron count rates with energy between 1 and $4 \mathrm{keV}$ on the $\mathrm{G} 1$ orbit which indicates changes in the mean location, or the surface-wave amplitude, or the thickness of the plasma sheet, or a combination of these.

Quasi-periodic modulations of energetic ion intensities in the Jovian magnetosphere were reported by Woch et al. (1998). These intensity variations are associated with changes of the particle energy spectra. Therefore to represent the modulations they used the spectral index $\gamma$. Typical periodicities of spectral index gamma are softening (higher index, steeper spectrum) during the plasma sheet thinning and abrupt hardening (lower index, flatter spectrum) during the plasma sheet dipolarization. Woch et al. (1998) observed modulations of the spectral index $\gamma$ for protons and ions of sulfur and oxygen with a time period which remains roughly constant at about 3 days on orbit $\mathrm{C} 9$ and $\mathrm{C} 10$ from about $20 R_{J}$ up to $145 R_{J}$ (see for oxygen panels (g) (C9) and (h) (C10) in Fig. 1, see also Table 1). These modulations were attributed to the quasi-periodic transition between two basic states of the Jovian magnetotail which recur with a time constant presumably inherent to the Jovian magnetosphere. The 


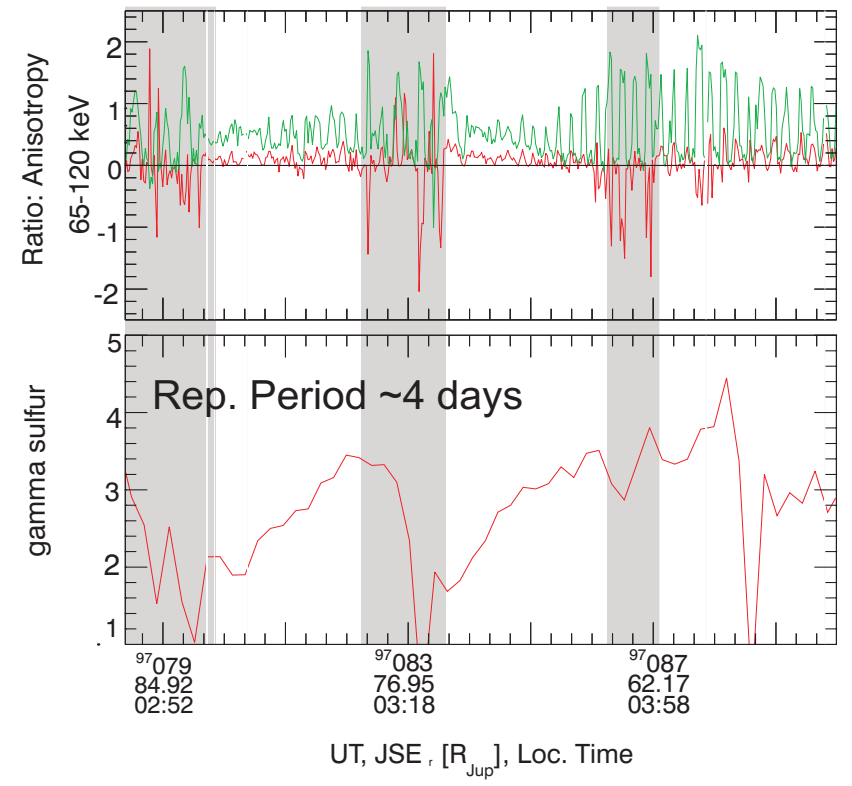

Fig. 2. First order anisotropies in the radial (red, positive is outward) and corotational direction (green) (top); the sulfur energy spectral index $\gamma$, averaged over 10-h-averages (bottom). The time interval DOY 078, 00:00 UT to DOY 090, 00:00 UT in 1997 (orbit E6) is shown. Grey shadowed areas indicate times at which the Jovian magnetotail is in a "disturbed" state. A "disturbed" state is usually associated with the ion flow bursts, i.e. deviations of the plasma flow from the corotational (the red component is higher than green).

first state is characterized by a thick plasma sheet with high intensities of energetic particles, a hard energy spectrum and the plasma flow in corotation direction. The second state is a thin plasma sheet with low intensities of energetic particles, a soft energy spectra and the plasma flow pattern associated with tailward and sunward flows.

Not only quasi-periodic modulations of the energy spectra and the intensities are observed on these orbits but also modulations of the relative ion abundance ratios, like $\mathrm{S} / \mathrm{O}, \mathrm{S} / \mathrm{He}$, $\mathrm{O} / \mathrm{He}$ and p/He (Radioti et al., 2007). The authors suggest that internal acceleration processes affect the ion composition in the whole magnetosphere.

After, the periodic modulations of the spectral index $\gamma$ (orbit E16 panel (f), in Fig. 1) were compared with variations observed in the magnetic field measurements, it was proposed that periodic stretching and dipolarization of the plasma sheet takes place in the Jovian magnetosphere with an intrinsic period of $\sim 3$ days (Kronberg et al., 2007).

Later, Kronberg et al. (2008a) discussed periodicities of particle flux, the magnetic field and spectral index $\gamma$ on Galileo orbits G8 and C9. These periodicities have been compared with a similar data set from the terrestrial magnetosphere and it was established to be similar to the periodic substorms at Earth in its morphology. The periodicity of the spectral index for helium on the G8 and C9 orbits is shown in Fig. 1, panel (i). It is observed at distances between $\sim 25$ and $\sim 130 R_{J}$ from the planet.

In addition to the particle data published we would like to add unpublished periodicities in the nightside of the Jovian magnetosphere. Periodicities of the spectral index $\gamma$ are observed in 1997 on E6 orbit (Day Of Year (DOY) 078-089) with a repetition period of 4-5 days (for sulfur, see panel $(\mathrm{k})$ in Fig. 1); orbit G7 (DOY 097-106), with a period of 2-3 days (for oxygen, see panel 1); orbit C10 (DOY 282-306) with a period of 3 days (for protons, see panel e); in 1998 orbit E18 (DOY 328-354) with a period of 2-7 days (for protons, see panel d); in 1999 orbit Io24 (DOY 292-305) with a period of 4 days (for sulfur, see panel a).

Not only the nightside of the Jovian magnetosphere is affected by periodic modulations of the plasma sheet structure. Similar periodicities are observed in the dayside as well. Consequently the periodic change of the plasma sheet structure is a global process, affecting the whole magnetosphere (Kronberg et al., 2007, 2008a). Examples are presented in Fig. 1, for orbits: G29 (DOY 000-011) (for oxygen, panel b) and Io33 (DOY 005-017) (for oxygen, panel c). Both examples show a repetition period of 3 days.

\subsection{Explosive events}

Whereas the above examples show more gradual variations, events of impulsive nature are reported from the nightside magnetosphere, namely the tail part of the dawn region. Ion flow bursts with a repetition period of 2-3 day were observed on the G2 orbit first by Krupp et al. (1998). They are a clear evidence of the active, explosively reconfigurating magnetotail. Later, Kronberg et al. (2005) showed that these bursts occur simultaneously with bipolar signatures in the magnetic field which are commonly considered to be a signature of plasmoids (Kronberg et al., 2008b). In the tail part of the magnetosphere and predominantly in the pre-dawn side particle flow bursts, which manifest the release of plasma are quite commonly observed. Kronberg et al. (2007) showed examples of particle bursts in the tail appearing after the plasma sheet becomes thin enough that the condition for an instability which can lead to reconnection is satisfied.

Further examples of observations of ion flow bursts are presented in Figs. 2 to 5. They also correspond to the black dots on the Galileo orbits shown in Fig. 1. During the ion flow bursts the typical corotational plasma flow changes its direction and becomes radially outward or inward, i.e. in Figs. 2 to 5 the first order anisotropy, radial component (red) dominates the azimuthal one (green). During the time periods presented in Figs. 2 and 5 when Galileo orbits were located planetward from the $\mathrm{x}$-line derived by Woch et al. (2002) mainly radially inward ion flow bursts are seen (negative radial anisotropy). They are mainly accompanied by a significant corotational component, that indicates a sunward moving plasma. The orbit G8 (Fig. 3) was very close 

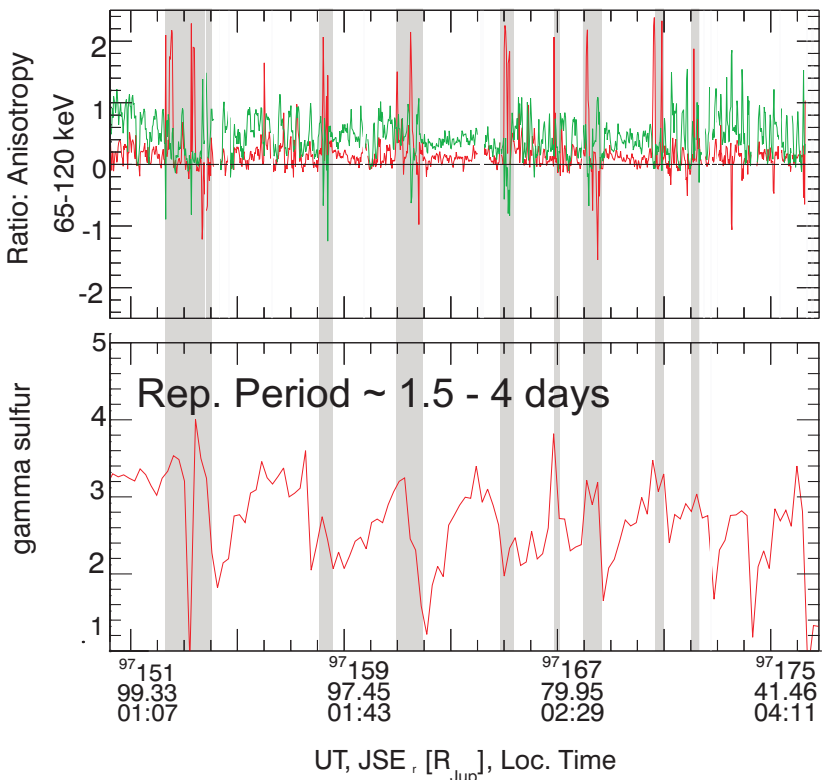

Fig. 3. Format as in Fig. 2. The time interval DOY 150, 00:00 UT to DOY 177, 00:00 UT in 1997 (orbit G8) is shown.

to the statistical $\mathrm{x}$-line and the orbit C9 (Fig. 4) was tailward from this $\mathrm{x}$-line. Therefore the mainly tailward bursts are observed and often followed by sunward bursts, implying that the $\mathrm{x}$-line is moving tailward. The bursts appear mainly after a significant thinning of the plasma sheet, when the spectral index $\gamma$ after a period of increase starts to decrease, i.e. between thinning and dipolarization (indicated by shaded bars in Figs. 2-5). The recurrent disruptions of the ambient particle flow in the corotational direction by radially outward/inward flow bursts are repetitive in nature with periods from 1.5 to 4 days. Especially nice examples are seen in Figs. 3-4.

In the dawn side the periodicities are less regular than in the dusk side as the ion bursts influence the shape of the spectra leading to the teeth-like spikes, see e.g. Fig. 1, panel (f) (at DOY 237, 246, 251), panel (i) (at DOY 153), panel (j) (at DOY 271, 288).

Moving further away from the planet, we can see in the deep tail a strong correlation between periodic bursts and periodic release of iogenic mass seen as flow speed variations (McComas et al., 2007), velocity dispersions, anisotropies and compositional variations (McNutt et al., 2007) in the observations made by particle instruments on board New Horizons in the distant tail $\left(600-1000 R_{J}\right)$. The variations have typical time scales of 3 to 4 days. The estimations from velocity dispersion provide evidence that events observed by $\mathrm{NH}$ are the same as observed by the EPD onboard Galileo. Also the composition of these energetic bursts is iogenic (Haggerty et al., 2009).
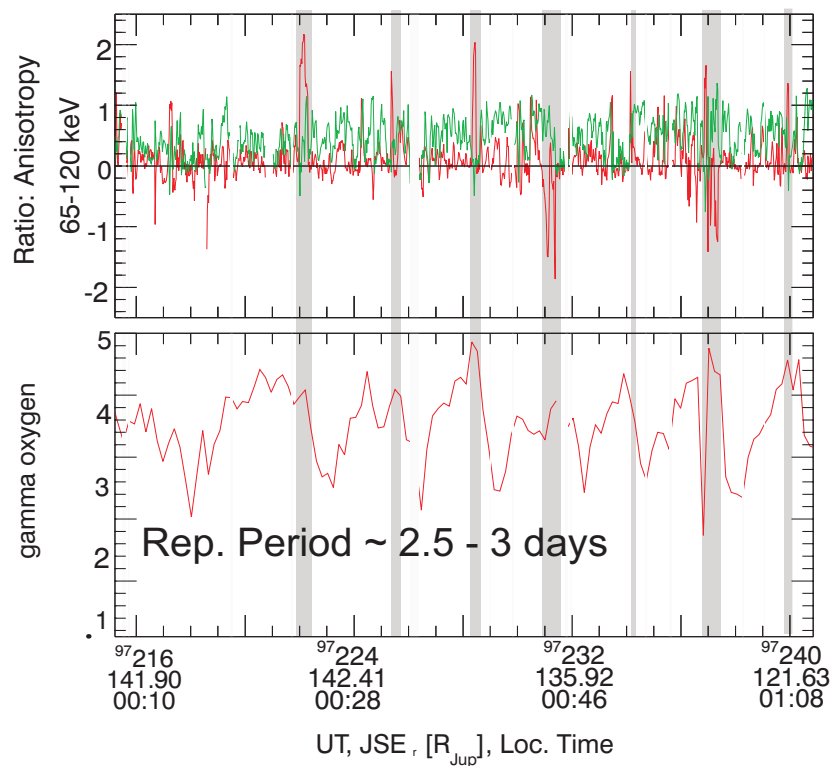

Fig. 4. Format as in Fig. 2. The time interval DOY 215, 00:00 UT to DOY 241, 00:00 UT in 1997 (orbit C9) is shown.

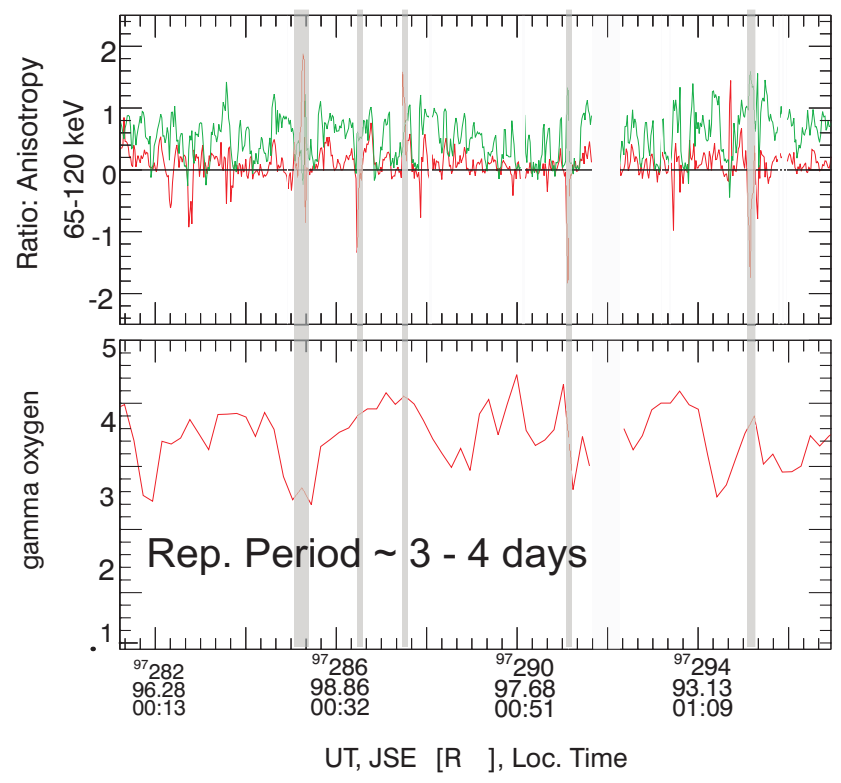

Fig. 5. Format as in Fig. 2. The time interval DOY 281, 00:00 UT to DOY 297, 00:00 UT in 1997 (orbit C10) is shown.

\subsection{Auroral dynamics}

The dynamical behavior of the Jovian magnetosphere also reflects in the auroral features. Observations made by the International Ultraviolet Explorer (IUE) showed periodicities of the "auroral activity index" which quantifies the variability of the flux radiated in the $\mathrm{H}_{2}$ bands (1560-1620 $\AA$ ) 
on scales of 5-10 days interval of 40 days (Prangé et al., 2001). The energy output in this spectral range is a direct measurement of the energy which the magnetosphere loses along auroral magnetic lines. These variations are very well correlated with the state (quiet or disturbed) of the magnetic field derived from Galileo observations along the spacecraft path at radial distances from $\sim 110$ to $10.7 R_{J}$. Therefore it was identified that some dynamical process affects the whole magnetosphere and triggers recurrent energy releases along auroral magnetic field lines. During the same time interval periodicities on scales of 7 days were observed by the plasma instrument (Vasyliūnas et al., 1997) and by EPD, both on Galileo (see panel $\mathrm{m}$ in Fig. 1 which shows the change of the spectral index $\gamma$ ). However we cannot directly correlate the auroral activity averaged over the whole auroral region with the variations of the magnetic field observed only in a specific magnetospheric sector.

Recent Hubble Space Telescope (HST) observations showed transient auroral features in the dawn region (polar dawn spots), with a 2-3 day quasi-periodic occurrence. Because of their mapped location and their periodic cycle, these polar spots were interpreted as signatures of internally driven magnetic reconnection in the Jovian magnetotail (Radioti et al., 2008).

Additionally, evidence that enhancements in the flux of various auroral radio emissions are systematically connected to energetic particles events is provided by observations with the Plasma Wave Subsystem (PWS) on Galileo orbits G2, E6, G7 and G8 (Louarn et al. (2000), see also Table 1). These events were interpreted as sudden energy releases in the Jovian magnetosphere. In most cases the events occur when the plasma sheet is thin and relatively depleted. A few hours after the events, Louarn et al. (2000) observe a thick and heavily populated plasma sheet. The authors point out that instead of the expected increase of the plasma density due to plasma loading from the moon Io before the events, they observed a low populated plasma sheet. Then the plasma evacuation process leads to a progressive decrease of the plasma sheet density and thickness as well. They suggest that an instability developing in the external part of the Io torus or in the near plasma sheet that sporadically injects new plasma populations to the more distant plasma sheet is responsible for the energetic events.

\section{Discussion}

\subsection{Essence from observations}

From the observations presented above we learned:

Quasi-periodic variations of the particles and fields properties are observed throughout the Jovian magnetosphere (from $20 R_{J}$ and further). Impulsive events are predominantly seen in the pre-dawn tail regions as well in the deep magnetotail (up to $\sim 1000 R_{J}$ ), gradual variations in most parts of the magnetosphere. Even in the dayside-magnetosphere we observe well defined periodicities (see Fig. 1).

The repetition period varies from $\sim 1.5$ to $\sim 7$ days. However most of the periodicities have periods in the range between 2.5 and 4 days. From the above presented overview we conclude that these periodicities are related to one complex dynamic process of the magnetospheric reconfiguration. The variation of spectral index $\gamma$ on longer time scales like ( $\sim 7$ days) observed on orbits E18 and G1 is similar to what we observed on most of the other trajectories. It can be a purpose of future studies to find out whether these deviations from the average period happen more often, or the definition of the repetition period is biased.

The periodic variations are most clearly seen in the iogenic ions, i.e. sulfur and oxygen. The periodic variations of protons and helium are also observed, although they are less pronounced. Quantitatively this can be expressed by an amplitude of the spectral index $\gamma$ : for sulfur and oxygen it changes from $\sim 2$ to $\sim 3.8$, i.e. $\Delta \gamma$ is $\sim 1.8$ when for helium and protons from $\sim 2$ to $\sim 3$, i.e. $\Delta$ is $\gamma \sim 1$. See also examples d, e and $i$ for protons and helium in Fig. 1. Radioti et al. (2007) (for the middle magnetosphere and near magnetotail, Galileo data) and Haggerty et al. (2009) (for the distant tail, NH data) taking into account the change in the ion composition concluded that periodic dynamics of the Jovian magnetosphere is influenced by ions from the internal source Io. Nevertheless as the periodicities are associated with the reconfiguration of the Jovian magnetosphere and according changes of its topology (like plasma sheet thickness) it is likely to expect periodicities for all ions.

\subsection{Relation to substorm}

The above observations of a process affecting the whole Jovian magnetosphere can be discussed in the framework of a magnetospheric substorm. We choose one concept of a substorm derived for the terrestrial case and will apply it to the Jovian magnetosphere.

A magnetospheric substorm basically consists of two phases. First mechanical stresses deform the magnetic field into a configuration of increased energy, then the magnetic configuration becomes unstable and rapidly releases the energy (Vasyliūnas, abstract for ICS-9). This process is associated with changes of the magnetic field topology and can be adapted to the Jovian magnetospheric system, replacing the solar wind stress by stress from rotationally driven outflow of iogenic plasma. Furthermore the main phenomena of a magnetospheric substorm at Earth were subsumed by Vasyliūnas (abstract for ICS-9) under three groups: (1) enhanced energy dissipation (auroral emissions) accompanied by (2) changes of the magnetic field configuration, from stretched to nearly dipolar, (3) fast (order of Alfvén speed or more) plasma bulk flows in the magnetotail.

We can apply this phenomenological grouping to Jupiter. The phenomena of group (1) were observed, e.g. by HST as 
periodic occurrence of polar emissions in the Jovian aurora (Radioti et al., 2008). Furthermore, Prangé et al. (2001) detected periodicities of the "auroral activity index" at somewhat longer time scales (5-10 days) which are correlated with magnetic field disturbances. Louarn et al. (1998) reported on periodic intensifications of the auroral emissions using in situ PWS data. They associated these with the change of the magnetic field topology and with particle bursts. Signatures from group 2 are represented by observations of the magnetic field change from a tail-like to a more dipolar configuration (Russell et al., 1998, 2000; Kronberg et al., 2007, 2008a). Also hints on periodic plasma sheet thinning and thickening are given by Vasyliūnas et al. (1997). In this paper we presented the periodic changes of the spectral index gamma on scales of 1.5-7 days associated with respective changes of the plasma sheet configuration throughout the whole magnetosphere. Eventually the periodic fast (order of Alfvén speed) plasma bulk flows in the magnetotail (group 3) were reported by Krupp et al. (2001); McComas et al. (2007), and by Kronberg et al. (2008b). Therefore we suggest that the phenomena belong to one single, global-scale process which can be named Jovian substorm.

Moreover, similar behavior of the spectral index is observed during periodic terrestrial substorms (Kronberg et al., 2008a). It is striking that these periodicities are also observed in the day side region. However, periodic thinning and thickening at all local times are also observed at the terrestrial magnetosphere during periodic substorms by Clauer et al. (2006).

\subsection{Driving mechanisms for the periodicity}

The excess of energy loaded to the terrestrial magnetosphere is released in periodic substorms accompanied by periodic thinning and thickening of the plasma sheet. At Earth the solar wind energy input could trigger the periodic substorm (Huang and Cai, 2009). Will the solar wind energy input lead to the periodical substorms at Jupiter?

Simulations by Fukazawa et al. $(2005,2006)$ show that during a purely northward Interplanetary Magnetic Field (IMF) the Jovian magnetosphere can reach an unstable state in which a nearly periodic series of magnetic $\mathrm{X}$ and $\mathrm{O}$ lines will be launched tailward. However, the purely northward IMF is observed less frequent than the periodicities which are observed at most Galileo trajectories.

Louarn et al. (2000) suggested that the periodic energetic events are triggered by sporadic plasma injections in the external part of Io torus or in the close magnetodisk. Their repetition period can vary depending on the density of the plasma sheet, the denser the plasma sheet is, the more often energetic events will be observed. The density variations could be connected with the solar wind conditions.

Southwood and Kivelson (2001) also considered the importance of the solar wind pressure influence on the dynamics of the Jovian magnetosphere. They expected to ob- serve magnetotail activity during low solar wind pressure, i.e. when the magnetosphere is expanded. In opposite, as inferred, for example by Tao et al. (2005), solar wind dynamic pressure enhancements can induce reconnection bursts in the Jovian magnetosphere. Therefore the general repetition period of the modulations set up by the mass release process can be reduced leading to deviations from the 3-4 day period. However as shown in Kronberg et al. (2008a) not every solar wind dynamic pressure enhancement leads to the reduction of the repetition period. In any case, the influence of the solar wind cannot be excluded and solar wind monitoring for future missions to Jupiter would be a great help.

Our interpretation of the energy source of the quasiperiodic reconfiguration process at Jupiter follows Woch et al. (1998). At Jupiter, excess energy is provided by an internal process, namely mass loading of rapidly rotating flux tubes from the moon Io. Mass loading and fast planetary rotation causing magnetotail field line stretching due to centrifugal forces. This leads to a magnetotail configuration favouring magnetic reconnection. Magnetic reconnection causes formation of plasmoids and release as well as dipolarization of field lines connected to the planet. Continued mass loading leads again to a stretching of the tail field lines. The quantitative model of Kronberg et al. (2007) based on this concept confirms this picture, yielding also time series of the repetition period close to the observed ones.

\subsection{Question on plasma circulation}

The models which describe the dynamics and circulation in the Jovian magnetosphere, e.g. the famous reconnectionrelated Vasyliūnas-cycle by Vasyliūnas (1983), the model by Cowley et al. (2003) who combined Vasyliūnas-cycle with the solar wind interaction Dungey-cycle, the model by Kivelson and Southwood (2005) who outlined the dynamics of the plasma sheet as a function of local time, do not consistently explain the periodic modulations of the plasma sheet. It would be an important task for the future to incorporate the periodic modulations in a more complex model.

\section{Conclusions and outlook}

In conclusion, we have summarized the observational evidences for periodic variations on time scales of 1.5 to 7 days of different physical quantities in the Jovian magnetosphere. Occasional observations of the change of the magnetospheric configuration with a repetition period 1.5 day as well as with repetition period 7 days reflect that the environment of the Jovian magnetosphere and internal processes are very dynamical and complex. The periodicities of the iogenic ions are more pronounced. The periodical thinning and thickening of the plasma sheet is observed at the nightside as well as at the dayside. We showed that these modulations are not an episodic and rare event but represent the signature of a global 
process which is an inherent feature of the Jovian magnetosphere. The modulations can be described by the two step concept of the terrestrial substorm. We conclude that the periodicity is internally driven but there are still many open questions.

The questions: How does the solar wind affect these periodic variations? Why are not they always observed? What are the factors influencing the repetition period? Whether the thinning (thickening) of the plasma sheet occur simultaneously in the dayside and the nightside or not? are still awaiting their answers. We are looking forward to future missions to Jupiter, as for example JMO which will help to gain more insight into the physical nature of this process. Having multipoint observations would be a great opportunity to reveal those questions.

Acknowledgements. We are thankful to Vytenis Vasyliūnas for stimulating of this work. Work at MPS was supported by the German Aerospace Centre (DLR) through contract $50 \mathrm{OH} 0801$ and by the Max Planck Gesellschaft.

Topical Editor R. Nakamura thanks two anonymous referees for their help in evaluating this paper.

\section{References}

Clauer, C. R., Cai, X., Welling, D., DeJong, A., and Henderson, M. G.: Characterizing the 18 April 2002 storm-time sawtooth events using ground magnetic data, J. Geophys. Res., 111, A04S90, doi:10.1029/2005JA011099, 2006.

Cowley, S. W. H., Bunce, E. J., Stallard, T. S., and Miller, S.: Jupiter's polar ionospheric flows: Theoretical interpretation, Geophys. Res. Lett., 30, 24-1, doi:10.1029/2002GL016030, 2003.

Fukazawa, K., Ogino, T., and Walker, R. J.: Dynamics of the Jovian magnetosphere for northward interplanetary magnetic field (IMF), Geophys. Res. Lett., 32, 3202, doi:10.1029/ 2004GL021392, 2005.

Fukazawa, K., Ogino, T., and Walker, R. J.: Configuration and dynamics of the Jovian magnetosphere, J. Geophys. Res., 111, 10207, doi:10.1029/2006JA011874, 2006.

Haggerty, D. K., Hill, M. E., McNutt, R. L., and Paranicas, C.: Composition of energetic particles in the Jovian magnetotail, J. Geophys. Res., 114, 2208, doi:10.1029/2008JA013659, 2009.

Huang, C. and Cai, X.: The magnetotail total pressure and lobe magnetic field at onsets of sawtooth events and their relation to the solar wind, J. Geophys. Res., 114, A13, doi:10.1029/2008JA013807, 2009.

Kivelson, M. G. and Southwood, D. J.: Dynamical consequences of two modes of centrifugal instability in Jupiter's outer magnetosphere, J. Geophys. Res., 110, A12209, doi:10.1029/ 2005JA011176, 2005.

Kronberg, E. A., Woch, J., Krupp, N., Lagg, A., Khurana, K. K., and Glassmeier, K.-H.: Mass release at Jupiter: Substorm-like processes in the Jovian magnetotail, J. Geophys. Res., 110, A03211, doi:10.1029/2004JA010777, 2005.

Kronberg, E. A., Glassmeier, K.-H., Woch, J., Krupp, N., Lagg, A., and Dougherty, M. K.: A possible intrinsic mechanism for the quasi-periodic dynamics of the Jovian magnetosphere, J. Geophys. Res., 112, A05203, doi:10.1029/2006JA011994, 2007.

Kronberg, E. A., , Woch, J., Krupp, N., Lagg, A., Daly, P. W., and Korth, A.: Comparison of periodic substorms at Jupiter and Earth, J. Geophys. Res., 113, A04212, doi:10.1029/ 2007JA012880, 2008a.

Kronberg, E. A., Woch, J., Krupp, N., and Lagg, A.: Mass release process in the Jovian magnetosphere: Statistics on particle burst parameters, J. Geophys. Res., 113, 10202, doi:10.1029/ 2008JA013332, 2008b.

Krupp, N., Woch, J., Lagg, A., Wilken, B., Livi, S., and Williams, D. J.: Energetic particle bursts in the predawn Jovian magnetotail, Geophys. Res. Lett., 25, 1249-1252, 1998.

Krupp, N., Lagg, A., Livi, S., Wilken, B., Woch, J., Roelof, E. C., and Williams, D. J.: Global flows of energetic ions in Jupiter's equatorial plane: First-order approximation, J. Geophys. Res., 106, 26017-26032, 2001.

Louarn, P., Roux, A., Perraut, S., Kurth, W., and Gurnett, D.: A study of the large-scale dynamics of the jovian magnetosphere using the Galileo plasma wave experiment, Geophys. Res. Lett., 25, 2905-2908, 1998.

Louarn, P., Roux, A., Perraut, S., Kurth, W. S., and Gurnett, D. A.: A study of the Jovian "energetic magnetospheric events" observed by Galileo: role in the radial plasma transport, J. Geophys. Res., 105, 13073-13088, doi:10.1029/1999JA900478, 2000.

McComas, D. J., Allegrini, F., Bagenal, F., Crary, F., Ebert, R. W., Elliott, H., Stern, A., and Valek, P.: Diverse Plasma Populations and Structures in Jupiter's Magnetotail, Science, 318, 217-220, doi:10.1126/science.1147393, 2007.

McNutt, R. L., Haggerty, D. K., Hill, M. E., Krimigis, S. M., Livi, S., Ho, G. C., Gurnee, R. S., Mauk, B. H., Mitchell, D. G., Roelof, E. C., McComas, D. J., Bagenal, F., Elliott, H. A., Brown, L. E., Kusterer, M., Vandegriff, J., Stern, S. A., Weaver, H. A., Spencer, J. R., and Moore, J. M.: Energetic Particles in the Jovian Magnetotail, Science, 318, 220-222, doi: 10.1126/science.1148025, 2007.

Prangé, R., Chagnon, G., Kivelson, M. G., Livengood, T. A., and Kurth, W.: Temporal monitoring of Jupiter's auroral activity with IUE during the Galileo mission. Implications for magnetospheric processes, Planet. Space Sci., 49, 405-415, 2001.

Radioti, A., Woch, J., Kronberg, E. A., Krupp, N., Lagg, A., Glassmeier, K.-H., and Dougherty, M. K.: Energetic ion composition during reconfiguration events in the Jovian magnetotail, J. Geophys. Res., 112, A06221, doi:10.1029/2006JA012047, 2007.

Radioti, A., Grodent, D., Gérard, J.-C., Bonfond, B., and Clarke, J. T.: Auroral polar dawn spots: Signatures of internally driven reconnection processes at Jupiter's magnetotail, Geophys. Res. Lett., 35, 3104, doi:10.1029/2007GL032460, 2008.

Russell, C. T., Khurana, K. K., Huddleston, D. E., and Kivelson, M. G.: Localized Reconnection in the Near Jovian Magnetotail, Science, 280, 1061-1064, 1998.

Russell, C. T., Khurana, K. K., Kivelson, M. G., and Huddleston, D. E.: Substorms at Jupiter: Galileo Observations of Transient Reconnection in the Near Tail, Adv. Space Res., 26, 1499-1504, 2000.

Southwood, D. J. and Kivelson, M. G.: A new perspective concerning the influence of the solar wind on the Jovian magnetosphere, J. Geophys. Res., 106, 6123-6130, 2001.

Tao, C., Kataoka, R., Fukunishi, H., Takahashi, Y., and Yokoyama, 
T.: Magnetic field variations in the Jovian magnetotail induced by solar wind dynamic pressure enhancements, J. Geophys. Res., 110, A11208, doi:10.1029/2004JA010959, 2005.

Vasyliūnas, V. M.: Plasma distribution and flow, Physics of the Jovian Magnetosphere, pp. 395-453, 1983.

Vasyliūnas, V. M., Frank, L. A., Ackerson, K. L., and Paterson, W. R.: Geometry of the plasma sheet in the midnight-to-dawn sector of the Jovian magnetosphere: Plasma observations with the Galileo spacecraft, Geophys. Res. Lett., 24, 869-872, 1997.
Woch, J., Krupp, N., Lagg, A., Wilken, B., Livi, S., and Williams, D. J.: Quasi-periodic modulations of the Jovian magnetotail, Geophys. Res. Lett., 25, 1253-1256, 1998.

Woch, J., Krupp, N., Khurana, K. K., Kivelson, M. G., Roux, A., Perraut, S., Louarn, P., Lagg, A., Williams, D. J., Livi, S., and Wilken, B.: Plasma sheet dynamics in the Jovian magnetotail: Signatures for substorm-like processes?, Geophys. Res. Lett., 26, 2137-2140, 1999.

Woch, J., Krupp, N., and Lagg, A.: Particle bursts in the Jovian magnetosphere: Evidence for a near-Jupiter neutral line, Geophys. Res. Lett., 29, 42-1, doi:10.1029/2001GL014080, 2002. 